\title{
Adult preventive vaccines with other synergistic lifestyle options: is it time to add these ancillary benefits to the overall AS management checklist?
}

\author{
Mark A. Moyad ${ }^{1}$
}

Received: 6 December 2020 / Accepted: 19 April 2021 / Published online: 7 May 2021

(c) The Author(s), under exclusive licence to Springer-Verlag GmbH Germany, part of Springer Nature 2021

\begin{abstract}
Purpose To review the potential ancillary cardiovascular and other health impacts of compliance with general adult vaccination series in the prostate cancer active surveillance (AS) population. No previous review has been published in regard to this specific topic.

Methods Literature review of PubMed data up to December 2020

Results Compliance rates for adult vaccination are in the approximate anemic range of 25-50\% with occasional higher rates of specific vaccines in the elderly population including annual influenza and pneumococcal prevention. Herpes zoster (HZ) and numerous other vaccine preventive illnesses are associated with an increased risk of cardiovascular events. Preliminary evidence suggests vaccine compliance could reduce overall morbidity and mortality, and adherence to heart healthy lifestyle changes and parameters could further improve vaccine efficacy and overall wellness. COVID-19 vaccine utilization and research should also continue to reinforce the direct and ancillary benefits of this entire preventive intervention category.

Conclusions Multiple ancillary lifestyle change recommendations could be included in the AS criteria to potentially reduce morbidity and mortality in this population, and perhaps the most unsung intervention is to improve the inadequate rates of general adult vaccination compliance and other heart healthy behavioral changes that impact their efficacy. Heart health, prostate health, and immune system health are closely interlinked.
\end{abstract}

Keywords Vaccines $\cdot$ Shingles $\cdot$ Active surveillance $\cdot$ Prostate cancer $\cdot$ Cardiovascular disease $\cdot$ Lifestyle changes $\cdot$ COVID19

\section{Introduction}

Active surveillance (AS) has been endorsed by multiple national and global clinical guidelines [1,2]. The ongoing robust medical literature on AS continues to reflect the enthusiasm for this management strategy. Select sub-topics within AS warrant greater awareness and attention. The primary cause of morbidity and mortality within most AS populations occur from cardiovascular disease (CVD) and other non-prostate cancer etiologies [3]. Thus, diverse lifestyle interventions to improve overall quality and/or longevity should be encouraged. Perhaps the behavioral change in need of the most immediate improvement is the rate of adult preventive vaccination compliance. Vaccination has both overt benefits and less well known potential ancillary advantages for AS patients. Healthy lifestyle changes have positive benefits on general health outcomes $[4,5]$. Lifestyle changes, including diet and exercise and other interventions, are synergistic with vaccine compliance and efficacy. This is a topic yet to receive adequate attention in the AS, prostate, and other cancer literature. Within this article, we hope to promote this approach with health care workers and AS patients.

Mark A. Moyad

moyad@med.umich.edu

1 Department of Urology, University of Michigan Medical Center, Ann Arbor, MI 48109-5330, USA 


\section{Cardiovascular events and vaccine compliance}

The prevention of cardiovascular events as a side benefit of adult vaccination could improve the overall health of AS patients. This holistic approach could also provide the needed impetus to improve overall general adult vaccination compliance rates, which unfortunately remain in the range of $25-50 \%$ [6, 7]. However, some specific vaccinations have greater compliance rates in the elderly population, such as those for influenza or pneumococcal prevention, while other critical vaccines such as Herpes zoster (HZ) or shingles continue to experience low compliance rates regardless of the qualifying age group. The importance of improving overall compliance with some emphasis on $\mathrm{HZ}$ vaccination cannot be overstated and again provides another potential paradigm of how health care professionals and patients could improve morbidity and mortality rates of AS patients.

\section{Shingles, cardiovascular events, and prostate cancer}

Varicella-zoster virus (VZV) is the infectious agent responsible for chickenpox (varicella) and HZ. Reactivation of VZV is responsible for $\mathrm{HZ}$, and its associated complications, which can cause considerable discomfort and other serious issues including any or some of the following: post-herpetic neuralgia (PHN), ocular involvement with the potential for temporary or even permanent vision loss, peripheral and/or cranial nerve palsies, Ramsay-Hunt syndrome, meningoencephalitis, disseminated disease, and the potential for early mortality [8-12]. One in three adults who have had varicella develop $\mathrm{HZ}$ in their lifetime. For example, estimates as high as $99 \%$ of individuals aged 40 and older in the U.S. harbor dormant VZV. Advanced age, comorbidities, psychological stress and family history are associated with an increased risk.

A prostate or other cancer diagnosis also appears to be associated with an elevated HZ risk [12]. Some prostate cancer treatments, including hormone therapy, appear to be preliminarily associated with an increased risk of $\mathrm{HZ}$ $[13,14]$. There have been case reports of men with prostate cancer impacted by $\mathrm{HZ}$ with subsequent increases in PSA levels at various stages of their disease, including AS $[15,16]$. It is of interest that treatment with antiviral medications in some of these situations resulted in PSA reductions. Thus, if men on AS are at an increased risk of $\mathrm{HZ}$ because of their age, cancer diagnosis, other comorbidities, or even family history of this condition it would appear imperative to raise vaccination awareness or compliance since men fall into at least one or more of these risk categories while on AS. Perhaps the additional increased risk of painful sequalae or serious morbidity from a bout of HZ, or even an increasing PSA would be enough to motivate AS clinicians and patients to inquire about vaccine status? How about the potential for an increased risk of cardiovascular events with a shingles diagnosis? Again, if cardiovascular disease events are the leading cause of mortality in AS participants, then potentially preventing such events via a preventive vaccine could be the sine qua non in not only discussing but tangibly improving adherence to adult vaccinations.

Observational research continues to suggest the acute and chronic inflammation resulting from shingles and other vaccine preventable infections could also create an abnormal vascular milieu, which could, at least temporarily, increase the risk of various cardiovascular events including myocardial infarction and stroke [17-22]. For example, the consistent clinical research suggestive of an increased risk of transient ischemic attack (TIA) or stroke with herpes zoster ophthalmicus (HZO) continues to imply the greater the severity of the infection then the subsequent greater risk of an ancillary cardiovascular event. Preventing shingles, as well as preventing the duration, or severity of this condition could result in subsequent cardiovascular event reductions from preliminary observational evidence [23-25].

The first shingles vaccine (Zostavax ${ }^{\circledR}$ ), available since 2006, is a zoster vaccine live (ZVL) attenuated product able to prevent $\mathrm{HZ}$ and some of its notable complications such as PHN, but the waning beneficial impacts with increasing age is one notable limitation of this product [26]. It reduces the risk of $\mathrm{HZ}$ by approximately $50 \%$ overall, but in those 70 years and older approximately $37 \%$. The inability to utilize this ZVL option for immune suppressed patients is another limitation. Still, a review of Medicare health records including more than 1,380,000 fee-for-service beneficiaries 66 years of age or older with no history of stroke and controlling for age, gender, race, medications and comorbidities found the potential for cardiovascular risk reduction benefits [23]. ZVL vaccine appeared to be associated with a reduction in ischemic and hemorrhagic stroke in the $60 \mathrm{~s}, 70$, and even above 80 age groups.

Since 2017, a novel adjuvanted, non-live recombinant zoster vaccine (RZV) known as "Shingrix" is available and should be discussed for patients on AS [26, 27]. The adjuvant itself is actually derived from a purified extract of the bark of the Chilean Soap Bark Tree (Quillaja saponaria) and contributes to the formidable $90-95 \%$ protection against shingles observed in populations 50 and older. One could argue it is a vaccine with a "natural" adjuvant that when utilized appropriately gives some of the most profound protection achieved for an adult preventive vaccine across a variety 
of age groups. A similar sourced "natural" adjuvant is being utilized currently in a phase 3 clinical trial of a COVID19 vaccine (Novavax, Inc, Gathersburg, MD) [28]. RZV requires a total of two intramuscular injections, each separated by 2-6 months. Vaccine efficacy in preventing PHN was approximately $89 \%$ in those 70 years and older. Cellular and humoral effects appear to last approximately a decade and potentially longer, but the novelty of this approach limits even longer-term data knowledge. Notable adverse reactions include temporary injection site pain in approximately $70-90 \%$ of patients ( $8-15 \%$ with placebo), and myalgia in $35-57 \%$ (10-15\% with placebo) [29]. Grade 3 adverse events occur in approximately less than $10 \%$. Older age groups reported less local and general adverse events.

The U.S. advisory committee on immunization practices (ACIP) recommend RZV as the standard or the first-line prophylactic vaccine for $\mathrm{HZ}$ and $\mathrm{PHN}$ prevention in those 50 years of age or older, regardless of previous ZVL status [30]. Canada also recommends RZV for similar age groups [31], and other countries are transitioning towards its utilization [32]. Thus, many AS patients will have received the initial ZVL and currently qualify for the RZV, as well as those having never received either vaccine, but have experienced recurrent shingles events. The potential ability to also utilize this option in some immune suppressed patients provides another major advantage. Patients should determine if they qualify with their clinician(s) since most AS patients will indeed be candidates to receive RZV, but it is the opinion of this author they should also be told of the potential ancillary benefits, and the potential for notable temporary adverse effects.

\section{Influenza, pneumonia, and other preventive vaccines and cardiovascular events}

A partial review of other general adult preventive vaccines suggests potential ancillary benefits abound. First the association between several different infections and cardiovascular events are becoming more established similar to the situation with HZ [33-35]. Multiple retrospective and prospective studies have demonstrated an association between influenza or pneumonia, disease severity, and an increased risk of a diverse range of cardiovascular events. Systemic infections produce a pro-inflammatory response, which can be prolonged in some patients, and increases the risk of vasculitis, cardiovascular disease progression, and even plaque rupture.

Preliminary data also suggest vaccination against influenza and pneumococcal pneumonia and a subsequent reduced risk of cardiovascular events, hospitalization and even all-cause mortality [35-37]. Interestingly, these diverse benefits also appear to occur in patients with prostate cancer [38], which is of interest since some observational studies suggest less attention toward cardiovascular health parameters post-prostate cancer diagnosis, and no notable increase in the general utilization of preventive vaccinations compared to the general population of similar age groups [39]. There is also the issue of health disparities among men with prostate cancer and the reduced access or utilization of preventive vaccinations [40], which urgently needs to be addressed to capitalize on these diverse benefits in diverse populations.

\section{COVID-19 cardiovascular disease paradigm and vaccine compliance?}

The current COVID-19 pandemic has directed profound attention toward the potential cardiovascular complications of an infectious systemic process, as well the additional negative prognostic impact of uncontrolled adverse cardiovascular parameters when dealing with systemic infections [41]. For example, arterial or venous thrombosis, myocardial injury and other cardiovascular complications have resulted from SARS-CoV-2 infections. It could be argued COVID-19 is both a respiratory and cardiovascular disease. Perhaps, COVID-19 will provide another notable example of when preventive methods are accessible then they should be considered not only for their direct but also ancillary potential benefits. The COVID-19 pandemic is a reminder of the importance of other chronic and infectious disease prevention advice and adherence within the AS population. An effective COVID-19 vaccination should not only prevent severe infection, but also cardiovascular events that would have arisen from this virus. It is already of interest surveys are suggesting COVID-19 vaccination would be more likely to be utilized by patients if their healthcare providers recommended or endorse vaccination against this specific infectious agent [42]. This should not be a surprise because it is similar to other behavioral choices when determining the utilization of other adult preventive vaccines. The unified endorsement from trusted health care professionals have positively and generally altered the preventive health landscape when change was needed [4]. For example, one relevant analogy is the improving smoking cessation rates, and perhaps even the eventual appreciation that tobacco exposure is not just replete with respiratory but cardiovascular and other health issues.

\section{Obesity, dietary randomized trials, and vaccines}

Excess weight or obesity has known detrimental impacts on all-cause morbidity and mortality, whether indices of body mass index (BMI) or waist circumference (WC) are utilized [43, 44]. This has pertinence within AS populations 
where borderline obesity appears to be the rule rather than the exception, which is a reflection of the general middleaged adult or even elderly population [45-47]. A recent randomized AS intervention trial known as MEAL (Men's Eating and Living study) demonstrated a mean baseline BMI of 28-29 with a median of 28 [47]. This dietary intervention clinical trial demonstrated no impact on prostate cancer progression over 2 years compared to control AS patients. However, there were also no significant between group differences on weight loss or other reliable cardiovascular parameters. Perhaps, a future goal with AS patients in clinical trials of lifestyle changes should be either an additional primary or even a secondary endpoint of cardiovascular parameters such as metabolic changes or clinically significant events.

The MEAL investigators were able to admirably and actively encourage healthy dietary changes and compliance in the intervention group and perhaps passively in the control arm [47]. The increased consumption of vegetables and caloric reductions of $-230 \mathrm{cal} /$ day over the first year and $-250 \mathrm{cal} /$ day over the entire 2-year trial in the intervention group were statistically significant vs controls. However, the control group also experienced mean reductions of approximately $-175 \mathrm{cal}$ in the first 12 months and $-120 \mathrm{cal}$ over the 2 years. Thus, one could argue that either given a greater caloric difference between groups, or simply more years to follow participants would have ultimately resulted in tangible cardiovascular changes or clinical significance via clinically meaningful weight loss differences. Again, at least the compliance with this dietary intervention also suggests the majority of the AS population is dedicated to ancillary healthy lifestyle changes when given instructional or educational opportunities. Arguably, holistic optimism in the AS population from the MEAL trial should supersede any disease-specific skepticism resulting from the initial 2-year prostate cancer progression results.

Perhaps additional perspective can be derived from past major breast cancer randomized trials involving lifestyle changes, which were designed, conducted, and initially published approximately 10-20 years before prostate cancer lifestyle randomized trials $[48,49]$. Significant weight loss over a 5-year period reduced the risk of aggressive breast cancer relapse after primary treatment in participants. In one of the largest breast cancer prevention randomized trials involving lifestyle changes, heart healthy benefits in the intervention group ultimately resulted in lower breast cancer incidence, but again favoring primarily a reduction in the risk of more aggressive sub-types [49]. Healthy cardiovascular changes appear to result in either improved morbidity and/or mortality statistics, and in regard to breast cancer a preliminary potential impact on reducing the risk of progression or appearance of aggressive disease. Again, perhaps prostate cancer could utilize this heart healthy first approach paradigm going forward to achieve tangible clinical heart healthy benefits when asking patients to adhere to notable lifestyle changes? It is the opinion of this author that breast or other cancer research did not necessarily have this initial heart healthy first foresight, but organically transformed into adopting this approach based on the results of these and other breast cancer randomized trials.

Weight loss or healthy weight management should be a primary goal of most AS populations for other less appreciated reasons. Past studies suggest multiple general preventive vaccines are less efficacious in obese adults [50,51]. For example, hepatitis A and B, influenza, and tetanus are just some of the vaccinations potentially hampered by excess weight, and theoretically the possibility of less efficacy with COVID vaccines are also plausible. Immunometabolism is a novel field researching the altered and suboptimal immune responses with weight increases. Chronic mild inflammation appears to cause impaired regulation, communication and perhaps even memory within immunological subsets. Obesity also increases the risk and severity of numerous vaccine preventable infections, and appears to prolong viral shedding, which creates concerning transmission risks and issues [52, 53]. Again, as the average or even median AS patient is currently overweight or borderline obese, similar to the general adult population, it would seem imperative to discuss the negative diverse consequences of harboring excess weight or waist in regard to cardiovascular and immune health.

\section{Miscellaneous lifestyle changes and vaccines}

The diverse and lengthy list of potential healthy lifestyle changes improving morbidity and mortality statistics are also interesting and continues to suggest multiple simultaneous or synergistic positive impacts on preventive vaccine compliance and efficacy [5]. Exercise reduces general anxiety, stress, and negative psychological mood, which have all been recognized to impact the perception and side effects of vaccination. Sub-optimal mental health factors, including stress, also appear to reduce physical activity compliance in prostate cancer patients [54], so mitigating this issue could provide a plethora of mental and physical health benefits. Improving diet, reducing alcohol exposure, smoking cessation, and improving sleep all have the potential to improve overall health, vaccine efficacy, and some of these lifestyle factors are also associated with a lower probability of aggressive cancers or maintaining differentiation $[5,55,56]$.

\section{Conclusion}

Vaccine preventive illness is a topic which has received minimal attention in the prostate cancer literature [4], including among AS participants. Perhaps, the impact of 
the COVID-19 pandemic will have an immediate influence on specific and general vaccination status within prostate and other cancers. The nexus between respiratory or other infections on cardiovascular outcomes has arguably become more solidified with the COVID-19 pandemic, as well as the potential plethora of side benefits with overall adult vaccination compliance. For example, tetanus toxoid is being investigated clinically in other cancers as a potential neoadjuvant option to enhance the impact of potential dendritic cell treatments [57]. Tetanus infection is also associated with cardiovascular instability. Hepatitis vaccinations for those that qualify have the ability to prevent hepatocellular cancer, which remains one of the most common causes of global cancer deaths [58]. The human papillomavirus (HPV), associated with cervical cancer, also increases the risk of anal, penile, vaginal, vulvar and oropharyngeal cancers, which is part of the recent decision to discuss this vaccine option not only in adult women, but also men aged 27-45 years [59]. The potential for side benefits with vaccine compliance, not only in the general population, but for other patient groups, especially AS should be discussed after the more overt benefits of these interventions are also covered. Again, this is a daunting task because of the general lack of awareness, past and current compliance rates in these age groups, and a host of other factors impeding progress that need to be addressed in the future from health disparities to other newly recognized issues such as living alone or without a partner [60]. Regardless, is it time to add lifestyle or behavioral advice including general vaccine compliance to the management checklist for AS patients? Yes! Perhaps the mantra of "Heart healthy is prostate healthy" should now be altered based on recent evidence to "heart healthy is prostate and immune system healthy" [4].

Acknowledgements The author is grateful to Dr. Laurence Klotz and all of the other wonderful individuals that assisted in making this unique active surveillance issue a reality.

Author contribution MAM: data collection, data analysis, manuscript writing/editing.

\section{Declarations}

Conflict of interest MA Moyad: no conflict of interest.

\section{References}

1. American Urological Association Clinically Localized Prostate Cancer: AUA/ASTRO/SUO Guideline. https://www.auanet.org/ guidelines/prostate-cancer-clinically-localized-(2017)

2. Mottet N, van den Bergh RCN, Briers E et al (2020) EAU-EANMESTRO-ESUR-SIOG guidelines on prostate cancer-2020 update. Part 1: screening, diagnosis, and local treatment with curative intent. Eur Urol. https://doi.org/10.1016/j.eururo.2020.09.042
3. Klotz L (2019) Contemporary approach to active surveillance for favorable risk prostate cancer. Asian J Urol 6:146-152. https://doi. org/10.1016/j.ajur.2018.12.003

4. Moyad MA (2020) Preventing lethal prostate cancer with diet, supplements, and Rx: heart healthy continues to be prostate healthy and "first do no harm" part III. Curr Urol Rep 21:22. https://doi.org/10.1007/s11934-020-00972-5

5. Zimmerman P, Curtis N (2019) Factors that influence the immune response to vaccination. Clin Microbiol Rev. https://doi.org/10. 1128/CMR.00084-18

6. Williams WW, Lu P-J, O'Halloran A et al (2017) Surveillance of vaccination coverage among adult populations-United States, 2015. MMWR Surveill Summ 66:1-28. https://doi.org/10.15585/ mmwr.ss6611a1

7. Darr AY, Gottfried S (2020) Identifying vaccination rates of adult patients in ambulatory care clinics. SAGE Open Med. https://doi. org/10.1177/2050312120935461

8. Vukelic D, Koncic DO, Prepolec J et al (2020) Clinical characteristics of hospitalized adults and adolescents with herpes zoster in Croatia: 20 years of a single-center experience. Croat Med J 61:401-409. https://doi.org/10.3325/cmj.2020.61.401

9. Marra F, Parhar K, Huang B et al (2020) Risk factors for herpes zoster infection: a meta-analysis. Open Forum Infectious Dis. https://doi.org/10.1093/ofid/ofaa005

10. Qian J, Heywood AE, Karki S et al (2019) Risk of herpes zoster prior to and following cancer diagnosis and treatment: a population-based prospective cohort. J Infect Dis 220:3-11. https://doi. org/10.1093/infdis/jiy625

11. Brown GR (1976) Herpes zoster: correlation of age, sex, distribution, neuralgia, and associated disorders. South Med J 69:576-578

12. Hansson E, Forbes HJ, Langan SM, Smeeth L, Bhaskaran K (2017) Herpes zoster risk after 21 specific cancers: populationbased case-control study. Br J Cancer 116:1643-1651. https://doi. org/10.1038/bjc.2017.124

13. Hsu CY, Chen PR, Chen HJ, Liang JA (2017) Association between radical prostatectomy and risk of herpes zoster. Eur J Clin Microbiol Infect Dis 36:337-342. https://doi.org/10.1007/ s10096-016-2806-6

14. Wu FJ, Kao LT, Sheu SY, Lin HC, Chung SD (2018) Increased risk of a herpes zoster attack in patients receiving androgen deprivation therapy for prostate cancer. Andrologia. https://doi.org/ 10.1111/and.12863

15. Jurhill RR, van der Veen H, van Leenders GJ, Verhagen PC (2009) Reduction of serum prostate-specific antigen levels following varicella-zoster infection and valaciclovir treatment in prostate cancer. Eur Urol 56:392-394. https://doi.org/10.1016/j.eururo.2009.02. 025

16. Mesci A, Liu SK, Loblaw DA (2018) Spurious elevation of prostate-specific antigen associated with shingles in a prostate cancer patient undergoing active surveillance. Clin Case Rep 6:23382340. https://doi.org/10.1002/ccr3.1838

17. Marra F, Ruckenstein J, Richardson K (2017) A meta-analysis of stroke risk following herpes zoster infection. BMC Infect Dis. https://doi.org/10.1186/s12879-017-2278-Z

18. Zhang Y, Luo G, Huang Y et al (2017) Risk of stroke/transient ischemic attack or myocardial infarction with herpes zoster: a systematic review and meta-analysis. J Stroke Cerebrovasc Dis 26:1807-1816. https://doi.org/10.1016/j.strokecerebrovasdis. 2017.04.013

19. Wu P-H, Chuang Y-S, Lin Y-T (2019) Does herpes zoster increase the risk of stroke and myocardial infarction? A comprehensive review. J Clin Med. https://doi.org/10.3390/jcm8040547

20. Patterson BJ, Rausch DA, Irwin DE et al (2019) Analysis of vascular event risk after herpes zoster from 2007 to 2014 US insurance claims data. Mayo Clin Proc 94:763-775. https://doi.org/10. 1016/j.mayocp.2018.12.025 
21. Kim J, Jeon J, Lee HS, Lee K-Y (2020) Association between the risk for cardiovascular events and antiviral treatment for herpes zoster. Clin Infect Dis. https://doi.org/10.1093/cid/ciaa1384

22. Piazza MF, Paganino C, Amicizia D et al (2020) The unknown health burden of herpes zoster hospitalizations: the effect on chronic disease course in adult patients $\geq 50$ years. Vaccines. https://doi.org/10.3390/vaccines8010020

23. Yang Q, Change A, Tong X, Merritt R (2020) American heart association. Abstract TP493 herpes zoster vaccine live and risk for stroke among medicare beneficiaries: population based matched cohort study. Stroke 51(Suppl 1):493

24. Klaric JS, Beltran TA, McClenathan BM (2019) An association between herpes zoster vaccination and stroke reduction among elderly individuals. Mil Med 184(Suppl 1):126-132. https://doi. org/10.1093/milmed/usy343

25. Totterdell J, Phillips A, Glover C et al (2020) Safety of live attenuated herpes zoster vaccine in adults 70-79 years: a self-controlled case series analysis using primary care data from Australia's MedicineInsight program. Vaccine 38:3968-3979. https://doi.org/10. 1016/j.vaccine.2020.03.054

26. Singh G, Song S, Choi E et al (2020) Recombinant zoster vaccine (Shingrix $\left.{ }^{\circledR}\right)$ : a new option for the prevention of herpes zoster and postherpetic neuralgia. Korean J Pain 33:201-207. https://doi.org/ 10.3344/kjp.2020.33.3.201

27. Heineman TC, Cunningham A, Levin M (2019) Understanding the immunology of Shingrix, a recombinant glycoprotein $\mathrm{E}$ adjuvanted herpes zoster vaccine. Curr Opin Immunol 59:42-48. https://doi.org/10.1016/j.coi.2019.02.009

28. Keech C, Albert G, Cho I et al (2020) Phase 1-2 trial of a SARS$\mathrm{CoV}-2$ recombinant spike protein nanoparticle vaccine. N Engl J Med. https://doi.org/10.1056/NEJMoa2026920

29. Shingrix Prescribing Information. https://www.fda.gov/media/ 108597/download

30. Dooling KL, Guo A, Patel M, et al (2018) Recommendations of the advisory committee on immunization practices for use of herpes zoster vaccines. MMWR Morb Mortal Wkly Rep 67:103-108. https://www.cdc.gov/mmwr/volumes/67/wr/mm6703a5.htm

31. Drolet M, Zhou Z, Sauvageau C et al (2019) Effectiveness and cost-effectiveness of vaccination against herpes zoster in Canada: a modelling study. CMAJ 191:E932-E939. https://doi.org/10. 1503/cmaj.190274

32. Jayasinghe S, Sheridan S, Macartney K (2020) Herpes zoster vaccination in Australia: what's available and who benefits? Aust Prescr 43:2-6. https://doi.org/10.18773/aust[rescr.2020.001

33. Gopal R, Marinelli MA, Alcorn JF (2020) Immune mechanisms in cardiovascular diseases associated with viral infection. Front Immunol. https://doi.org/10.3389/fimmu.2020.570681

34. Mohammad MA, Tham J, Koul S et al (2020) Association of acute myocardial infarction with influenza: a nationwide observational study. PLoS One https. https://doi.org/10.1371/journal. pone.0236866

35. Fountoulaki K, Tsiodras S, Polyzogopoulou E et al (2018) Beneficial effects of vaccination on cardiovascular events: myocardial infarction, stroke, heart failure. Cardiology 141:98-106. https:// doi.org/10.1159/000493572

36. Cheng Y, Cao X, Cao Z et al (2020) Effects of influenza vaccination on the risk of cardiovascular and respiratory diseases and all-cause mortality. Ageing Res Rev. https://doi.org/10.1016/j.arr. 2020.101124

37. Pang $\mathrm{Y}$, Wang $\mathrm{Q}, \mathrm{Lv}$ M et al (2020) Influenza vaccination protects against hospitalization outcomes among older patients with cardiovascular or respiratory diseases. J Infect Dis. https://doi.org/ 10.1093/infdis/jiaa493

38. Li C-Y, Chen L-C, Lin H-Y et al (2020) Impact of 23-valent pneumococcal polysaccharide vaccination on the frequency of pneumonia-related hospitalization and survival in elderly patients with prostate cancer: a seven-year nationwide matched cohort study. Cancer. https://doi.org/10.1002/cncr.33203

39. Wallner LP, Slezak JM, Loo RK et al (2017) Ten-year trends in preventive service use before and after prostate cancer diagnosis: a comparison with noncancer controls. Perm J 21:16-184. https:// doi.org/10.7812/TPP/16-184

40. Li J, Thompson TD, Richards TB et al (2016) Racial and ethnic differences in health behaviors and preventive health services among prostate cancer survivors in the United States. Prev Chronic Dis. https://doi.org/10.5888/pcd13.160148

41. Madjid M, Safavi-Naeini P, Solomon SD, Vardeny O (2020) Potential effects of coronaviruses on the cardiovascular system: a review. JAMA Cardiol 5:831-840. https://doi.org/10.1001/jamac ardio.2020.1286

42. Reiter PL, Pennell ML, Katz ML (2020) Acceptability of a COVID-19 vaccine among adults in the United States: how many people would get vaccinated? Vaccine 38:6500-6507. https://doi. org/10.1016/j.vaccine.2020.08.043

43. Chen C, Ye Y, Zhang Y et al (2019) Weight change across adulthood in relation to all cause and cause specific mortality: prospective cohort study. BMJ. https://doi.org/10.1136/bmj.15584

44. Jayedi A, Soltani S, Zargar MS et al (2020) Central fatness and risk of all cause mortality: systematic review and dose-response meta-analysis of 72 prospective cohort studies. BMJ. https://doi. org/10.1136/bmj.m3324

45. Greenberg JW, Leinwand G, Feibus AH et al (2020) Prospective observational study of a racially diverse group of men on active surveillance for prostate cancer. Urology. https://doi.org/ 10.1016/j.urology.2020.09.044

46. Gregg JR, Surasi DS, Childs A et al (2020) The association of periprostatic fat and grade group progression in men with localized prostate cancer on active surveillance. J Urol. https://doi.org/ 10.1097/JU0000000000001321

47. Parson JK, Zahrieh D, Mohler JL et al (2020) Effect of a behavioral intervention to increase vegetable consumption on cancer progression among men with early-stage prostate cancer: the MEAL randomized clinical trial. JAMA 323:140-148. https://doi.org/10. 1001/jama.2019.20207

48. Chlebowski RT, Blackburn GL, Thomson CA et al (2006) Dietary fat reduction and breast cancer outcome: interim efficacy results from the women's intervention nutrition study. J Natl Cancer Inst 98:1767-1776. https://doi.org/10.1093/jnci/djj494

49. Chlebowski RT, Aragaki AK, Anderson GL, et al. on behalf of the Women's Health Initiative (2020) Dietary modification and breast cancer mortality: long-term follow-up of the women's health initiative randomized trial. J Clin Oncol 38:1419-1428. https://doi. org/10.1200/jco.19.00435

50. Painter SD, Ovsyannikova IG, Poland GA (2015) The weight of obesity on the human immune response to vaccination. Vaccine 33:4422-4429. https://doi.org/10.1016/j.vaccine.2015.06.101

51. Tagliabue C, Principi N, Giavoli C, Esposito S (2016) Obesity: impact of infections and response to vaccines. Eur J Clin Microbiol Infect Dis 35:325-331. https://doi.org/10.1007/ s10096-015-2558-8

52. Maier H, Lopez R, Sanchez N et al (2018) Obesity increases the duration of influenza A virus shedding in adults. J Infect Dis 218:1372-1382. https://doi.org/10.1093/infdis/jiy370

53. Luzi L, Radaelli MG (2020) Influenza and obesity: its odd relationship and the lessons for COVID-19 pandemic. Acta Diabetol. https://doi.org/10.1007/s00592-020-01522-8

54. Halbert CH, Jefferson MS, Drake R et al (2021) Social and clinical determinants of physical activity in prostate cancer survivors. Support Care Cancer 29:459-465. https://doi.org/10.1007/ s00520-020-05482-1

55. Ziouziou I, Touzani AM, Lahlou L et al (2020) Association of prostate cancer with nuts, seeds, alcohol and processed meats: a 
worldwide population-based study. Nutr Cancer. https://doi.org/ 10.1080/01635581.2020.1841250

56. Foerster B, Pozo C, Abufaraj M et al (2018) Association of smoking status with recurrence, metastasis, and mortality among patients with localized prostate cancer undergoing prostatectomy or radiotherapy: a systematic review and meta-analysis. JAMA Oncol 4:953-961. https://doi.org/10.1001/jamaoncol.2018.1071

57. Eoli M, Corbetta C, Anghileri E et al (2019) Expansion of effector and memory $\mathrm{T}$ cells is associated with increased survival in recurrent glioblastomas treated with dendritic cell immunotherapy. Neurooncol Adv. https://doi.org/10.1093/noajn1/vdz022

58. Chang M-H (2021) Prevention of hepatitis b virus infection and liver cancer. Recent Results Cancer Res 217:71-90. https://doi. org/10.1007/978-3-030-57362-1_4

59. Meites E, Szilagyi PG, Chesson HW et al (2019) Human papillomavirus vaccination for adults: updated recommendations of the advisory committee on immunization practices. MMWR Morb Mortal Wkly Rep 68:698-702. https://doi.org/10.15585/mmwr. mm6832a3

60. Jain A, van Hoek AJ, Boccia D, Thomas SL (2017) Lower vaccine uptake amongst older individuals living alone: a systematic review and meta-analysis of social determinants of vaccine uptake. Vaccine 35:2315-2328. https://doi.org/10.1016/j.vaccine.2017.03.013

Publisher's Note Springer Nature remains neutral with regard to jurisdictional claims in published maps and institutional affiliations. 